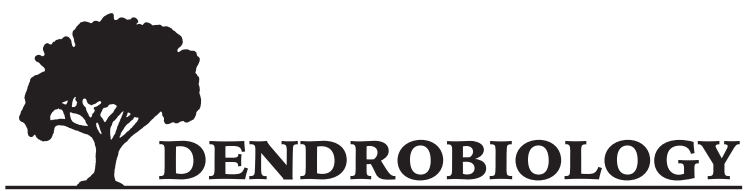 \\ 2013, vol. 69, 87-97
}

\author{
Patrycja Golińska, Hanna Dahm
}

\section{Antagonistic properties of Streptomyces isolated from forest soils against fungal pathogens of pine seedlings}

Received: 27 July 2012; Accepted 17 October 2012

\begin{abstract}
Biological control of plant diseases has received worldwide attention in recent years mainly as a response to use of hazardous chemicals in the environment. Soil actinomycetes particularly Streptomyces spp. enhance soil fertility and have antagonistic activity against wide range of soil-borne plant pathogens. In study of antifungal activity of streptomycetes against plant fungal-pathogens Fusarium oxysporum (Schlecht.), F. culmorum (Wm. G. Sm.) Sacc. and Rhizoctonia solani (Kühn) causing damping-off of pine seedlings, in vitro and in vivo tests were carried out. Two types of in vitro tests in mixed cultures of actinomycetes and fungi were performed. In majority of the cases, an inhibitory effect of actinomycetes on fungi was noticed. Three isolates (SG9, SR2, SR4) were highly active against pathogenic fungi and were chosen for exopolysaccharides extraction and in vivo tests. The sensitivity to exopolysaccharide action was noted in fungi from the genus Fusarium. Fusarium oxysporum showed a higher sensitivity to the analyzed exopolysaccharides than F. culmorum. Biocontrol interactions both in sterile and non-sterile soil were found for the actinomycete Streptomyces cyaneus (strain SG9) isolated from root free soil of the pine. The latest experiment confirmed the strong pathogenicity of the fungus Rhizoctonia solani.

Antifungal properties of Streptomyces cyaneus (strain SG9) need further comprehensive studies.
\end{abstract}

Additional key words: Streptomyces sp.; antifungal activity, Fusarium oxysporum, F. culmorum, Rhizoctonia solani, biocontrol activity

Address: P. Golińska, H. Dahm, Department of Microbiology, Nicolaus Copernicus University, Faculty of Biology and Environmental Protection, Lwowska 1, 87-100 Toruń, Poland, e-mail: golinska@umk.pl

\section{Introduction}

Gram-positive bacteria from the genus Streptomyces are one of the basic components of soil microorganisms. Actinomycetes of cultivated soils are well known and are still being intensively investigated. Less attention has been paid to actinomycetes of forest soils as acid forest soils are not believed to favor the development of these microorganisms (Barabasz and Vořišek 2002; Marcinowska 2002; Zakalyukina et al. 2002; Aghighi et al. 2004; Zakalyukina et al. 2004).
Members of the genus Streptomyces are important in nature mainly because of their high enzymatic activity. They actively participate in the biodegradation of various soil polymers both of plant and animal origin in the form of polysaccharides (starch, pectin, cellulose, chitin), proteins, including keratin and elastin which are difficult to degrade, and lignocelluloses and aromatic compounds (McCarthy 1987; Crawford 1988; Lechevalier 1988; McCarthy and Williams 1992; Marcinowska 1993; Kämpfer 2006). Due to their hydrolytic abilities and high adaptive 
capability they are an important link in the circulation of matter and energy (Dahm et al. 1986; Marcinowska 1993).

Over one-half of microorganisms isolated from soil show antagonistic properties. Among them actinomycetes are predominant, mainly from the genera Streptomyces and Micromonospora (Marcinowska 1993; Marcinowska 2002; Shirokikh et al. 2002). The production of bioactive metabolites which are secreted and accumulated in the cells and their effects on other microorganisms, mainly pathogenic ones, still arouses scientific and social interest (Marcinowska 2002; Shirokikh et al. 2002). One of the matters arousing an increasing interest among various scientific groups, especially plant pathologists, is biocontrol. New microorganisms are constantly being sought which could be used for combating fungal pathogens, the main causes of plant diseases (Aghighi et al. 2004).

Members of the genus Streptomyces show activity which can be used for the biocontrol of fungal pathogens of plants (Chattopadhyay and Nandi 1982; Fravel and Roberts 1991; Whipps 2001; de Boer et al. 2003; Dong et al. 2003; Kucuk and Kivanc 2003). This includes the production of various secondary metabolites (e.g. antibiotics), extracellular polysaccharides (EPS) and enzymes hydrolyzing fungal cell walls (Aghighi et al. 2004; Gohar et al. 2006).

In Central Europe the main causes of seedlings damping-off are fungi from the genus Fusarium (F. oxysporum, F. culmorum) and Rhizoctonia solani. Damping-off is a dangerous disease in forest nurseries and it is a burden in artificial renewal sowing and self-sowing. Fungi from the species Rhizoctonia solani are strong plant pathogens and cause damping-off of seedlings, especially in coniferous trees, including the Scots pine. Fusarium oxysporum also attacks pine seedling roots, mainly their root collar (Mańka 1992).

The purpose of the investigations was to show antagonistic properties of forest soil actinomycetes against dangerous fungal pathogens of pine seedlings and the selection of strains with potential biocontrol properties.

\section{Materials and Methods}

\section{Bacterial and fungal strains}

The microbial material analyzed were actinomycetes isolated from bulk soil (10 strains) and the rhizosphere (10 strains) of Scots pine (Pinus sylvestris L.) and pathogenic fungi causing damping-off of pine seedlings. The strains of actinomycetes were isolated from 87-year old Scots pine trees stand in the Forestry Bielawy near Toruń, Poland (Lat 53 $01^{\prime} 46^{\prime \prime}$; Long $\left.18^{\circ} 42^{\prime} 24^{\prime \prime}\right)$ and were identified up to species by Golińska and Dahm (2011b) according to the meth- ods described by Shirling and Gottlieb (1966), Kutzner (1981) and Williams et al. (1983). Strains used for the investigations:

- From the bulk soil: Streptomyces griseoviridis (SG1, SG6, SG8), Streptomyces cyaneus (SG2, SG4, SG9), Streptomyces varsoviensis (SG5), Streptomyces exfoliatus (SG7), Streptomyces chromogenus (SG10) and Streptoverticillium olivovertici (SG3).

- From the rhizosphere: Streptomyces exfoliatus (SR1, SR5, SR8, SR9, SR10), S. xanthochromogenes (SR2), Streptomyces phaeochromogenes (SR3), Streptomyces anulatus (SR4), Streptomyces griseoviridis (SR7) and Streptoverticillium olivovertici (SR6).

- Pathogenic fungi: Fusarium oxysporum (Schlecht.), Fusarium culmorum (Wm. G. Sm.) Sacc. and Rhizoctonia solani (Kühn) (strain no. 13, 15, 411 and 476). Because of the large strain differentiation of Rhizoctonia solani four strains of this species were used for investigations.

\section{Antagonism of Streptomyces strains against pathogenic fungi - in vitro tests}

These investigations were performed in mixed cultures of actinomycetes and fungi in Petri dishes with PDA medium (Difco) according to methods in Yuan and Crawford (1995) and Aghighi et al. (2004).

In Yuan and Crawford (1995) the results of mutual interactions between the actinomycetes and the fungus were observed after 5 days of incubation. The radius of the fungal colony in the fungal control culture and mixed cultures with actinomycetes were measured. The degree of antagonism of actinomycetes against fungi was expressed as an index of \% inhibition which was calculated according to the formula:

$\%$ inhibition $=(1$ - (fungal radius in a mixed culture with actinomycetes / fungal radius in control colony) $\times 100$ (Coombs et al. 2004).

In Aghighi et al. (2004) the size of inhibition zones formed in fungal cultures [mm] was measured.

On the basis of the obtained results, three highly antagonistic actinomycete strains (SG9, SR2, SR4) were chosen for further investigations.

\section{Antifungal activity of exopolysaccharides (EPS) isolated from selected Streptomyces strains}

In order to select optimal culture conditions for actinomycete growth, microorganisms were grown in 3 liquid media with different compositions (M1, M2 and M3) according to Gohar et al. (2006).

Exopolysaccharides were extracted from supernatant of actinomycetes culture broth according to Gohar et al. (2006).

Investigations on the effects of EPS derived from actinomycetes against pathogenic fungi were performed on Petri dishes with PDA medium (Difco) us- 
ing two-layer plates method modified from Aghighi et al. (2004). After inoculation of the basal layer of the PDA medium with a pathogenic fungus, filter paper discs (5 $\mathrm{mm}$ in diameter) were placed on the surface of the top agar layer. Each disc received $20 \mu \mathrm{l}$ of the aqueous EPS solution. Antagonistic activity of extracted EPS was examined in triplicates. The cultures were maintained at $26^{\circ} \mathrm{C}$ for 7 days after which fungal inhibition zones $[\mathrm{mm}]$ were measured.

\section{Biocontrol activity of Streptomyces strains - in vivo tests}

Pine seedlings were grown from certified seeds obtained from the forest nursery in Klosnowo near Chojnice, Poland.

The culture medium was soil from under Scots pine, taken from a stand from which actinomycetes had previously been isolated. Cultures of seedlings were performed in two variants - in non-sterile and sterile soil. Sterile soil was obtained by sterilizing 3 times in an autoclave at $121^{\circ} \mathrm{C}$ for 20 minutes.

The seedlings were grown in a culture chamber at $22 \pm 4^{\circ} \mathrm{C}$ in the light of sodium lamps with an illumination of 7 thousand lux (appr.100 $\mu \mathrm{mol} \mathrm{m}^{-2} \mathrm{~s}^{-1} \mathrm{PAR}-$ photosynthetically active radiation -16 hours and 8 hours in the dark).

For seedling inoculation a suspension of actinomycete spores in peat with sand prepared according to the method of Yuan and Crawford (1995) was used.

Fungi (Fusarium oxysporum, F. culmorum, Rhizoctonia solani) were grown in liquid Czapek-Dox medium (Difco). After three days of culture with shaking a suspension for seedling inoculation was prepared. The culture was centrifuged in a centrifuge for 10 minutes at $3500 \cdot \mathrm{g}$. The mycelium was suspended in $300 \mathrm{ml}$ sterile distilled $\mathrm{H}_{2} \mathrm{O}$ and homogenized. Subsequently $1 \mathrm{ml}$ inoculum was used to inoculate three-week old pine seedlings.

As controls seedlings not inoculated with any microorganism or inoculated only with the appropriate fungus or actinomycetes were used. The test seedlings were inoculated with two microorganisms - an appropriate fungus and an actinomycete. The experiments were performed in both the sterile and the non-sterile soil medium. Each variant of the experiment was performed in 10 replicates.

After 14 weeks duration of the experiment, seedling analysis was performed and the following measurements were taken: main root length $(\mathrm{cm})$, fresh root mass (mg), dry root mass (mg), shoot length $(\mathrm{cm})$, fresh shoot mass $(\mathrm{mg})$, dry shoot mass $(\mathrm{mg})$.

\section{Results}

\section{Antagonistic activity of streptomyces strains isolated from the rhizosphere and bulk soil of Scots pine against pathogenic fungi - in vitro tests}

Analysis of antagonistic interactions of actinomycetes against fungal pathogens of pine determined by the degree (\%) of fungal growth inhibition in mixed cultures indicated that in the majority of cases, an inhibitory effect of actinomycetes was seen on fungi. However, in some cases a stimulation of fungal growth was observed, e.g. stimulation of Fusarium oxysporum by the Streptomyces exfoliatus (SR8) strain. Stimulation of the growth of this fungus was as high as $159 \%$ (Fig. 1B). For the same fungus a small stimulation of growth (1-9.8\%) by Streptomyces varsoviensis (SG5), S. exfoliatus (SG7), S. cyaneus (SG9) and S. griseoviridis (SR7) was observed. Similar results were obtained for the culture of Fusarium culmorum and Streptomyces cyaneus (SG4) (Fig. 1A).

Actinomycetes showed the highest antagonism towards Fusarium culmorum and strains of Rhizoctonia solani. Antagonism was demonstrated both by soil actinomycetes [Streptoverticillium olivovertici (SG3), Streptomyces varsoviensis (SG5), S. exfoliatus (SG7), S. griseoviridis (SG8), S. cyaneus (SG9)], as well as rhizospheric ones [S. xanthochromogenes (SR2), S. phaeochromogenes (SR3), S. anulatus (SR4)]. The degree of fungal growth inhibition was $40-50 \%$. The strongest antagonists of Fusarium oxysporum growth were strains belonging to the species Streptomyces griseoviridis (strains SG1 and SG6) and a strain of Streptomyces exfoliatus (strain SR5), which inhibited fungal growth by $40 \%$ (Fig. 1B, Phot. 1). The four strains of Rhizoctonia solani $(13,15,411,476)$ analyzed in this work differed in their sensitivity to interaction of actinomycetes (Fig. 1C-F). No effects on the analyzed strains of $R$. solani were found for two Streptomyces cyaneus strains (SG2 and SG4), Streptomyces chromogenus (SG10) and Streptomyces exfoliatus (SR8).

It was observed that actinomycete strains belonging to the same species differed in the degree of fungal inhibition and thus the fungal inhibition was actinomycete strain property. Among three strains of Streptomyces cyaneus (SG2, SG4, SG9) only one (SG9) showed a high antagonism against fungi. The remaining strains did not affect fungal growth or only had a small influence. Strains of Streptomyces exfoliatus isolated from the rhizosphere (SR1, SR5, SR8, SR9, SR10) were very weak antagonists, whereas strain (SG7), of the same species derived from root free soil inhibited pathogen growth by $40-50 \%$.

Among actinomycetes isolated from the bulk soil, more strains antagonistic to fungi were observed than among strains isolated from the rhizosphere. Fusarium 

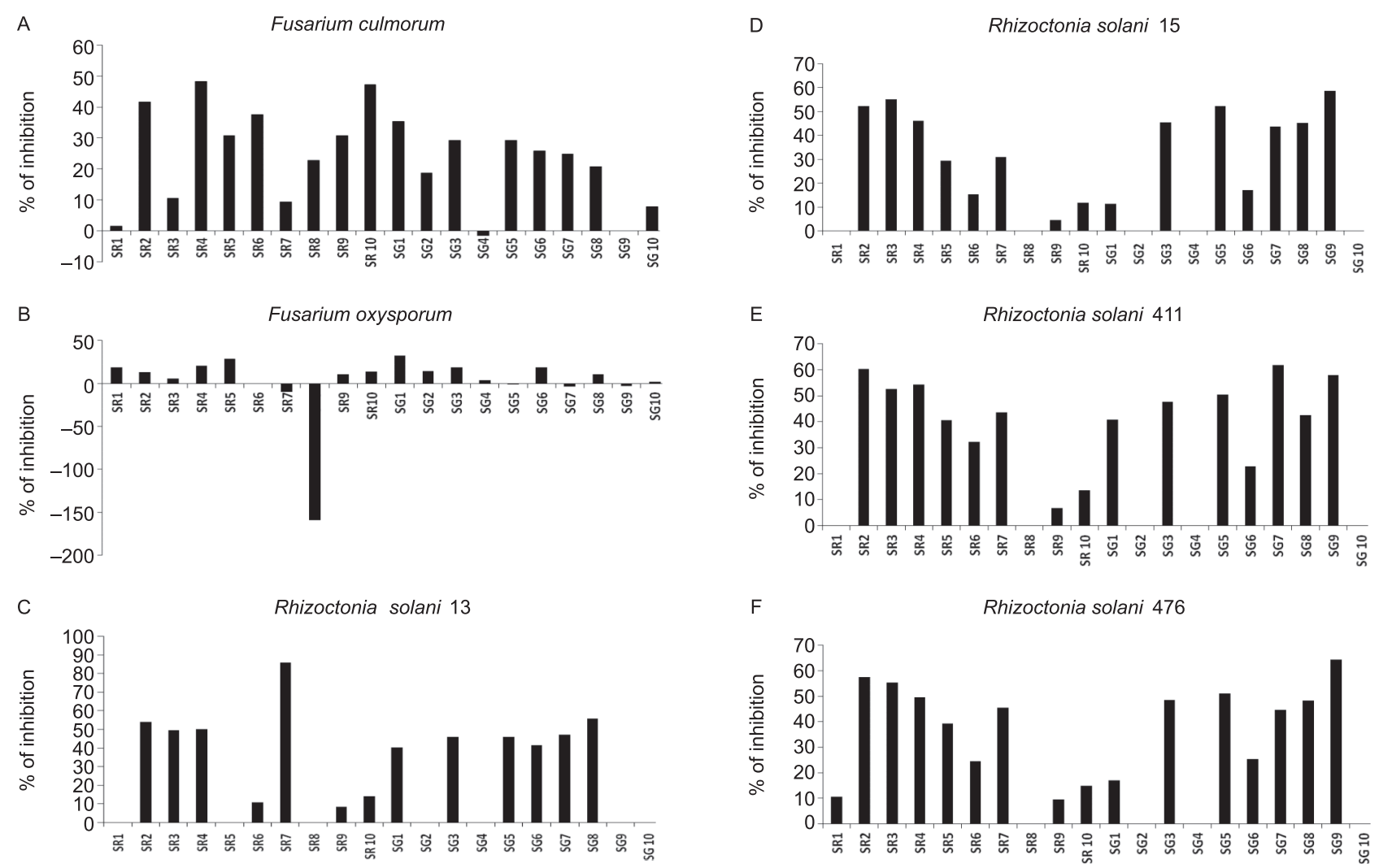

Fig. 1. Antagonism Streptomyces strains isolated from rhizosphere and bulk soil of Scots pine (Pinus sylvestris L.) against pathogenic fungi according to Yuan and Crawford method (1995) (fungi were inoculated five days after Streptomyces)

SR1-SR10 - Streptomyces strains isolated from rhizosphere of Scots pine; SG1-SG10 - Streptomyces strains isolated from bulk soil of Scots pine

oxysporum showed low sensitivity to actinomycetes inhibition. Even though the inhibition of growth of this fungus was observed in the presence of almost all actinomycetes, the degree of this inhibition was lower than for the remaining fungi. The growth of another species from this genus, Fusarium culmorum, was inhibited by 18 of the 20 analyzed strains of actino-

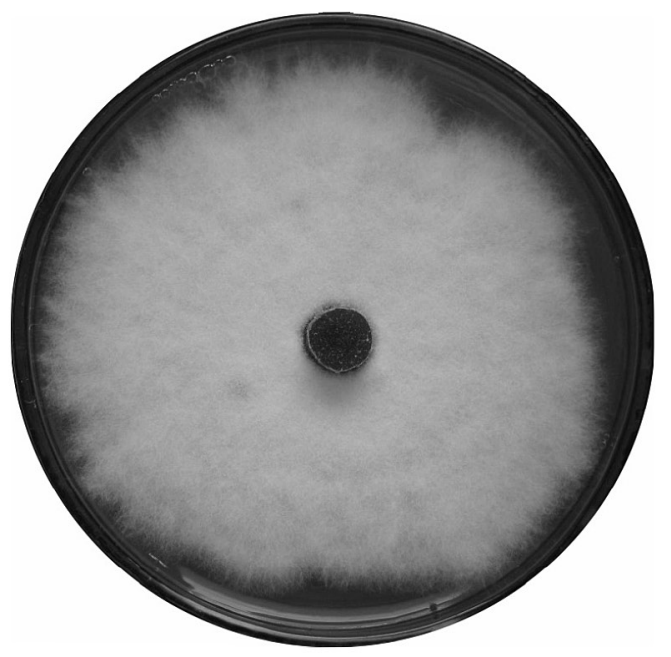

Fusarium oxysporum (Control) mycetes. The degree of inhibition of the growth of this fungus was differentiated.

After using an alternative method of analysis according to Aghighi et al. (2004), conclusions as to inhibition of fungi were made on the basis of clear zones of growth inhibition or of zones (which were not clear) of poorer fungal growth around an actino-

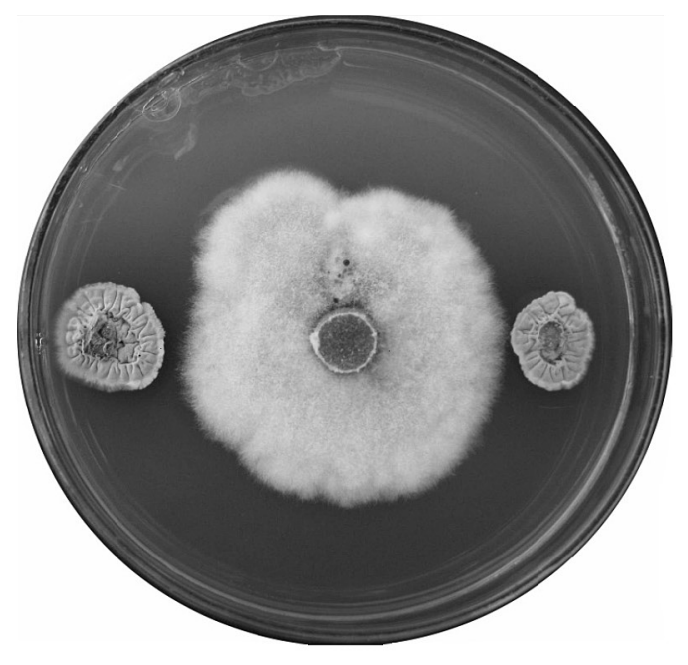

F. oxysporum + S. griseoviridis (SG1)

Fot. 1. Antagonistic activity of Streptomyces isolated from rhizosphere and bulk soil of Scots pine against pathogenic fungi according to Yuan and Crawford (1996) method (an example)

SG1 - Streptomyces griseoviridis isolated from bulk soil of Scots pine 
A

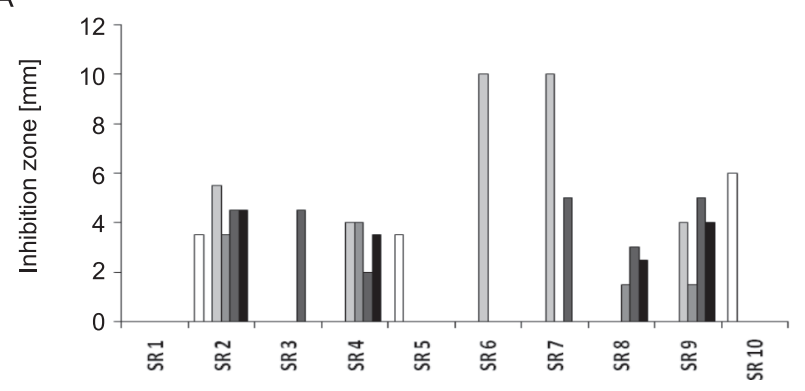

B

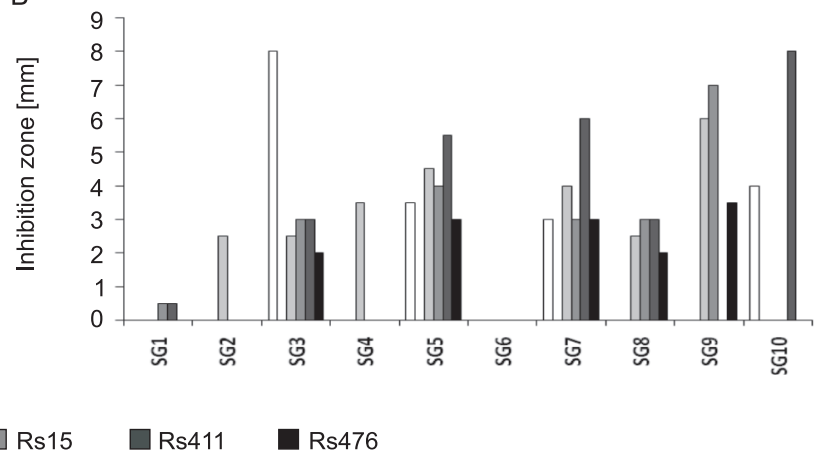

Fig. 2. Antagonism Streptomyces strains isolated from rhizosphere and bulk soil of Scots pine (Pinus sylvestris L.) against pathogenic fungi according to Aghighi et al. (2004) method (Streptomyces and fungi were inoculated simultaneously) SR1-SR10 - Streptomyces strains isolated from rhizosphere of Scots pine; SG1-SG10 - Streptomyces strains isolated from bulk soil of Scots pine; FC - Fusarium culmorum; FO - Fusarium oxysporum; Rs13 - Rhizoctonia solani 13; Rs15 - Rhizoctonia solani15;

Rs 411 - Rhizoctonia solani 411; Rs 476 - Rhizoctonia solani 476

mycetes colony. Soil and rhizospheric actinomycetes inhibited the growth of Rhizoctonia solani (3 among 4 strains) most strongly with clear zones of inhibition. The strongest antagonists of pathogenic fungi were soil actinomycetes (Streptoverticillium olivovertici (SG3), Streptomyces varsoviensis (SG5), S. exfoliatus (SG7), S. griseoviridis (SG8)) and in lower numbers rhizosphere actinomycetes (Streptomyces anulatus (SR4), Streptomyces exfoliatus (SR9)) (Fig. 2A, 2B, Phot. 2).
Inhibitory properties of EPS (third experimental variant) were deduced on the basis of clear zones of growth inhibition or zones of decreased fungal growth around a filter paper disc containing suspension of the extracted exopolysaccharide. The sensitivity to exopolysaccharide activity was noted in fungi from the genus Fusarium. No effect of exopolysaccharides on the growth of Rhizoctonia solani strains was observed.

Fusarium oxysporum showed a higher sensitivity to the analyzed exopolysaccharides than Fusarium

Table 1. Antagonistic activity of exopolysaccharides (EPS) isolated from Streptomyces strains against pathogenic fungi (growth inhibition or fungistatic zone $[\mathrm{mm}]$ )

\begin{tabular}{cccc}
\hline EPS source & Fusarium culmorum & Fusarium oxysporum & EPS g/ml \\
\hline SR2 & $8.2^{*}$ & $17^{* *}$ & 0.40 \\
SR4 & not inhibited & not inhibited & 0.84 \\
SG9 & $2.5^{* *}$ & $7.8^{*}$ & 0.23 \\
\hline
\end{tabular}

Rhizoctonia solani strains were not inhibited by studied exopolysaccharides

SR2 - Streptomyces xanthochromogenes; SR4 - Streptomyces anulatus isolated from rhizosphere of Scots pine; SG9 - Streptomyces cyaneus isolated from bulk soil of Scots pine; *Fungistatic activity (decreased fungal growth); **Inhibition zone

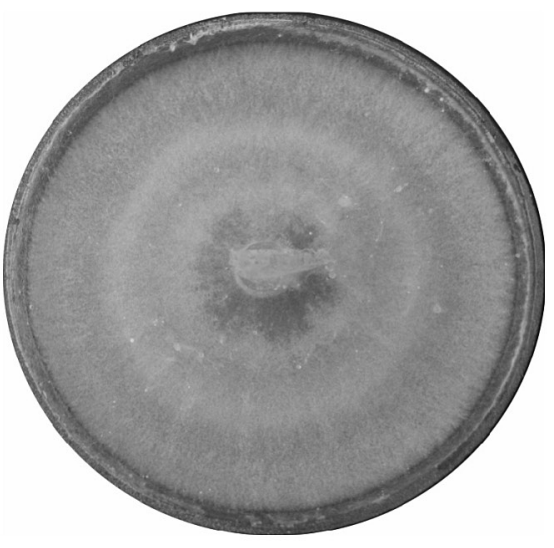

R. solani (Control)

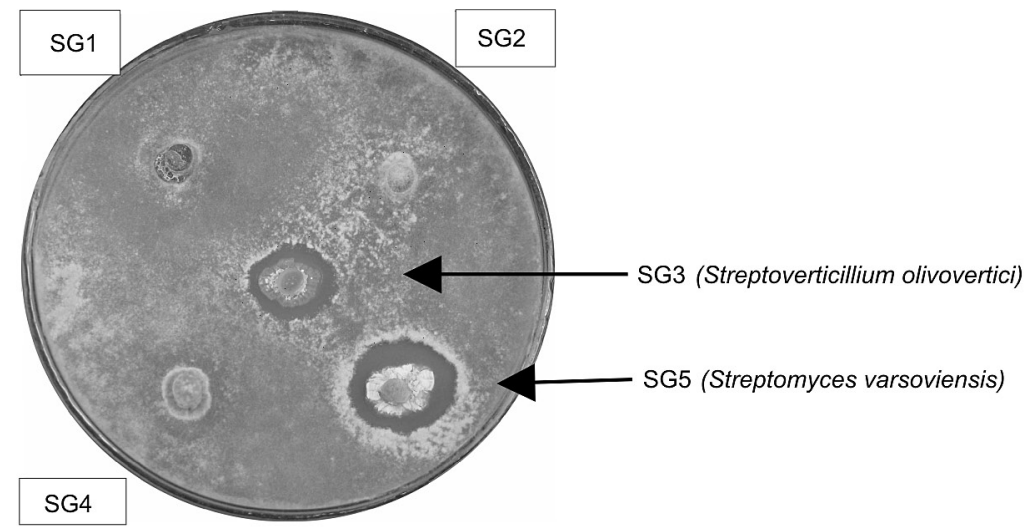

R. solani+streptomyces (SG1-SG5)

Fot. 2. Antagonistic activity of Streptomyces strains isolated from rhizosphere and bulk soil of Scot pine against pathogenic fungi according to Aghighi et al. (2004) method (an example)

SG1-SG5 Streptomyces strains isolated from bulk soil of Scots pine 


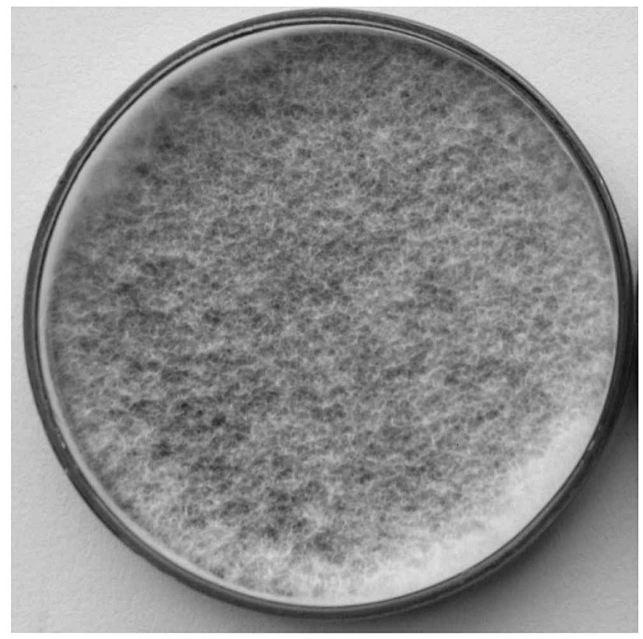

Fusarium oxysporum (Control)

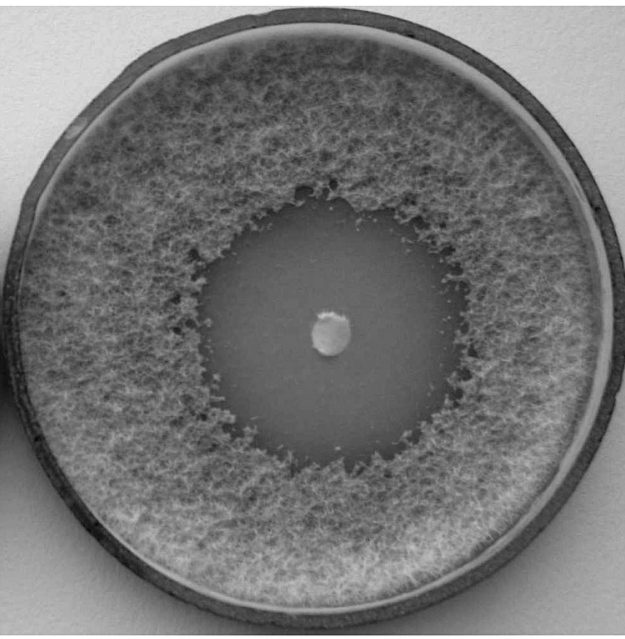

EPS (SR2) + Fusarium oxysporum

Fot. 3. Antagonistic activity of exopolysaccharides (EPS) isolated from Streptomyces xanthochromogenes (SR2) against Fusarium oxysporum (an example)

SR2 - Streptomyces xanthochromogenes isolated from rhizosphere of Scots pine

Table 2. Influence of Streptomyces strains on the growth of Scots pine seedlings after inoculation of Rhizoctonia solani [average values $(n=10) \pm$ standard deviations]

\begin{tabular}{|c|c|c|c|c|c|}
\hline \multicolumn{6}{|c|}{ Sterile soil } \\
\hline \multirow[b]{2}{*}{ Experimental combinations } & \multirow{2}{*}{$\begin{array}{l}\text { Number of dead } \\
\text { seedlings }\end{array}$} & \multicolumn{2}{|c|}{ Root } & \multicolumn{2}{|c|}{ Shoot } \\
\hline & & $\begin{array}{l}\text { Length of main root } \\
{[\mathrm{cm}]}\end{array}$ & $\begin{array}{l}\text { Dry mass } \\
{[\mathrm{mg}]}\end{array}$ & $\begin{array}{l}\text { Length } \\
{[\mathrm{cm}]}\end{array}$ & $\begin{array}{c}\text { Dry mass } \\
{[\mathrm{mg}]}\end{array}$ \\
\hline \multirow[t]{3}{*}{ Rhizoctonia solani - control } & 7 & 6,38 & 26,14 & 3,36 & 48,48 \\
\hline & & $\pm 9,0$ & $\pm 39,0$ & $\pm 2,1$ & $\pm 69,7$ \\
\hline & & $100 \%$ & $100 \%$ & $100 \%$ & $100 \%$ \\
\hline \multirow[t]{3}{*}{ R. solani + SR2 } & 4 & 13,15 & 45,70 & 3,37 & 46,16 \\
\hline & & $\pm 10,8$ & $\pm 47,1$ & $\pm 1,49$ & $\pm 38,2$ \\
\hline & & $206,11 \%$ & $174,82 \%$ & $100,29 \%$ & $95,21 \%$ \\
\hline \multirow[t]{3}{*}{ R. solani + SR4 } & 7 & 5,95 & 44,25 & 2,66 & 41,37 \\
\hline & & $\pm 7,5$ & $\pm 71,2$ & $\pm 2,1$ & $\pm 63,0$ \\
\hline & & $93,26 \%$ & $169,28 \%$ & $79,16 \%$ & $85,33 \%$ \\
\hline \multirow[t]{3}{*}{ R. solani + SG9 } & 2 & 14,21 & 52,90 & 4,20 & 55,29 \\
\hline & & $\pm 9,0^{*}$ & $\pm 39,0$ & $\pm 2,1$ & $\pm 69,7$ \\
\hline & & $222,72 \%$ & $202,37 \%$ & $125 \%$ & $114,04 \%$ \\
\hline \multicolumn{6}{|c|}{ Non-sterile soil } \\
\hline \multirow[b]{2}{*}{ Experimental combinations } & \multirow[b]{2}{*}{$\begin{array}{l}\text { Number of dead } \\
\text { seedlings }\end{array}$} & \multicolumn{2}{|c|}{ Root } & \multicolumn{2}{|c|}{ Shoot } \\
\hline & & $\begin{array}{l}\text { Length of main root } \\
{[\mathrm{cm}]}\end{array}$ & $\begin{array}{l}\text { Dry mass } \\
{[\mathrm{mg}]}\end{array}$ & $\begin{array}{l}\text { Length } \\
{[\mathrm{cm}]}\end{array}$ & $\begin{array}{c}\text { Dry mass } \\
{[\mathrm{mg}]}\end{array}$ \\
\hline \multirow[t]{3}{*}{ Rhizoctonia solani - control } & 7 & 7,14 & 18,89 & 3,35 & 49,82 \\
\hline & & $\pm 8,3$ & $\pm 29,3$ & $\pm 2,1$ & $\pm 73,8$ \\
\hline & & $100 \%$ & $100 \%$ & $100 \%$ & $100 \%$ \\
\hline \multirow[t]{3}{*}{ R. solani $+\mathrm{SR} 2$} & 2 & 16,26 & 27,88 & 5,56 & 112,09 \\
\hline & & $\pm 8,4^{*}$ & $\pm 22,8$ & $\pm 2,1^{*}$ & $\pm 80,56$ \\
\hline & & $227,73 \%$ & $147,59 \%$ & $165,97 \%$ & $224,99 \%$ \\
\hline \multirow[t]{3}{*}{ R. solani + SR4 } & 3 & 13,89 & 34,64 & 4,99 & 138,64 \\
\hline & & $\pm 8,5$ & $\pm 26,1$ & $\pm 2,6$ & $\pm 102,6^{*}$ \\
\hline & & $194,53 \%$ & $183,87 \%$ & $148,95 \%$ & $278,28 \%$ \\
\hline \multirow{3}{*}{ R. solani + SG9 } & 1 & 17,14 & 50,77 & 5,39 & 128,87 \\
\hline & & $\pm 6,1^{*}$ & $\pm 24,1^{*}$ & $\pm 2,4$ & $\pm 60,2^{*}$ \\
\hline & & $240,05 \%$ & $268,76 \%$ & $160,89 \%$ & $258,67 \%$ \\
\hline
\end{tabular}

SR2 - Streptomyces xanthochromogenes; SR 4 - Streptomyces anulatus isolated from rhizosphere of Scots pine; SG9 - Streptomyces cyaneus isolated from bulk soil of Scots pine; * Significant differences as compared to control (t-test) 
Table 3. Three-factor analysis of variance (ANOVA), comparing the kind of growth medium, streptomyces inoculation and pathogenic fungi inoculation on the growth parameters of Scots pine seedlings roots

\begin{tabular}{|c|c|c|c|c|c|}
\hline \multirow{2}{*}{ Source of variation } & \multicolumn{5}{|c|}{$p$ (significance level) } \\
\hline & $\mathrm{df}$ & Length of root & \multicolumn{2}{|c|}{ Fresh mass of root } & Dry mass of root \\
\hline Medium & 1 & 0,1021 & \multicolumn{2}{|c|}{0,0000} & 0,0000 \\
\hline Streptomyces & 3 & 0,0071 & \multicolumn{2}{|c|}{0,1135} & 0,0309 \\
\hline Pathogenic fungi & 2 & 0,0000 & \multicolumn{2}{|c|}{0,0000} & 0,0000 \\
\hline \multicolumn{6}{|c|}{ Newman-Keuls multiple range test $(p \leq 0,05)$} \\
\hline Soil: & Length of root $[\mathrm{cm}]$ & \multicolumn{2}{|c|}{ Fresh mass of root $[\mathrm{mg}]$} & \multicolumn{2}{|c|}{ Dry mass of root [mg] } \\
\hline sterile & $16,0066 \mathrm{a}$ & \multicolumn{2}{|c|}{292,4792 b } & \multicolumn{2}{|c|}{ sterylne: 80,0683 b } \\
\hline non-sterile & $17,1200 \mathrm{a}$ & \multicolumn{2}{|c|}{152,3508 a } & \multicolumn{2}{|c|}{ niesterylne: 43,5266 a } \\
\hline \multirow[t]{5}{*}{ Streptomyces } & Control: 14,8833 a & \multicolumn{2}{|c|}{ Control: 225,9333 a } & \multicolumn{2}{|c|}{ Control: 57,8900 a } \\
\hline & SR2: $17,5983 \mathrm{~b}$ & \multicolumn{2}{|c|}{ SR2: $208,0283 \mathrm{a}$} & \multicolumn{2}{|c|}{ SR2: $55,9866 \mathrm{a}$} \\
\hline & SR4: $15,9916 \mathrm{ab}$ & \multicolumn{2}{|c|}{ SR4: 251,4617 a } & \multicolumn{2}{|c|}{ SR4: 71,9766 b } \\
\hline & SG9: $17,7800 \mathrm{~b}$ & \multicolumn{2}{|c|}{ SG9: 204,2367 a } & \multicolumn{2}{|c|}{ SG9: $61,3366 \mathrm{ab}$} \\
\hline & Control: $18,6700 \mathrm{~b}$ & \multicolumn{2}{|c|}{ Control: 272,1675 b } & \multicolumn{2}{|c|}{ Control: 79,3162 c } \\
\hline \multirow[t]{2}{*}{ Pathogenic fungi } & F. oxysporum: $19,2550 \mathrm{~b}$ & \multicolumn{2}{|c|}{ F. oxysporum: 261,5237 b } & \multicolumn{2}{|c|}{ F. oxysporum: 68,4300 b } \\
\hline & R. solani: $11,7650 \mathrm{a}$ & \multicolumn{2}{|c|}{ R. solani: 133,5538 a } & \multicolumn{2}{|c|}{ R. solani: 37,6462 a } \\
\hline
\end{tabular}

df - degrees of freedom; SR2 - Streptomyces xanthochromogenes; SR4 - Streptomyces anulatus isolated from rhizosphere of Scots pine; SG9 Streptomyces cyaneus isolated from bulk soil of Scots pine; mean values marked with the same letter do not differ significantly $(p \leq 0,05)$

culmorum. The strongest action inhibiting the growth of $F$. oxysporum was noted for EPS obtained from strain Streptomyces xanthochromogenes (SR2) isolated from the rhizosphere of Scots pine (Phot. 3). The exopolysaccharide also inhibited growth of Fusarium culmorum causing the formation of non-clear zones of growth inhibition, but this action was weaker in comparison to that observed in F. oxysporum. EPS obtained from strain Streptomyces anulatus (SR4), even though it was tested at a concentration of two times higher
$(0.84 \mathrm{~g} / \mathrm{ml})$ than the EPS from strain SR2 and four times higher than that from the strain SG9, did not inhibit the growth of any of the tested fungi (Table 1).

\section{Biocontrol activity of streptomyces isolated from the rhizosphere and bulk soil of Scots pine against pathogenic fungi - in vivo tests}

The experiment confirmed the strong pathogenicity of the fungus Rhizoctonia solani. Seedlings inocu-

Table 4. 3 Three-factor analysis of variance (ANOVA), comparing the kind of growth medium, streptomyces inoculation and pathogenic fungi inoculation on the growth parameters of seedlings of Scots pine shoots

\begin{tabular}{|c|c|c|c|c|c|}
\hline \multirow{2}{*}{ Source of variation } & \multicolumn{5}{|c|}{$p$ (significance level) } \\
\hline & $\mathrm{df}$ & Length of shoot & \multicolumn{2}{|c|}{ Fresh mass of shoot } & Dry mass of shoot \\
\hline Medium & 1 & 0,0000 & \multicolumn{2}{|c|}{0,0000} & 0,0000 \\
\hline Streptomyces & 3 & 0,1109 & \multicolumn{2}{|c|}{0,1121} & 0,2089 \\
\hline Pathogenic fungi & 2 & 0,0000 & \multicolumn{2}{|c|}{0,0000} & 0,0265 \\
\hline \multicolumn{6}{|c|}{ Newman-Keuls multiple range test $(p \leq 0,05)$} \\
\hline Soil & Length of shoot $[\mathrm{cm}]$ & \multicolumn{2}{|c|}{ Fresh mass of shoot $[\mathrm{mg}]$} & \multicolumn{2}{|c|}{ Dry mass of shoot $[\mathrm{mg}]$} \\
\hline sterile & 4,8058 a & \multicolumn{2}{|c|}{299,3983 a } & \multicolumn{2}{|c|}{88,3288 a } \\
\hline non-sterile & $5,8358 \mathrm{~b}$ & \multicolumn{2}{|c|}{540,4517 b } & \multicolumn{2}{|c|}{$152,597 \mathrm{~b}$} \\
\hline \multirow[t]{5}{*}{ Streptomyces } & Control: 4,9500 a & \multicolumn{2}{|c|}{ Control: 391,4600 a } & \multicolumn{2}{|c|}{ Control: $109,8900 \mathrm{a}$} \\
\hline & SR2: $5,3430 \mathrm{a}$ & \multicolumn{2}{|c|}{ SR2: 410,5083 a } & \multicolumn{2}{|c|}{ SR2: $115,5000 \mathrm{a}$} \\
\hline & SR4: $5,4500 \mathrm{a}$ & \multicolumn{2}{|c|}{ SR4: $485,2100 \mathrm{~b}$} & \multicolumn{2}{|c|}{ SR4: 138,6583 a } \\
\hline & SG9: 5,5200 a & \multicolumn{2}{|c|}{ SG9: 392,5217 a } & \multicolumn{2}{|c|}{ SG9: 107,2317 a } \\
\hline & Control: 5,8387 b & \multicolumn{2}{|c|}{ Control: 467,6537 b } & \multicolumn{2}{|c|}{ Control: 128,4867 b } \\
\hline \multirow[t]{2}{*}{ Pathogenic fungi } & F. oxysporum: $6,0137 \mathrm{~b}$ & \multicolumn{2}{|c|}{ F. oxysporum: $519,6550 \mathrm{~b}$} & \multicolumn{2}{|c|}{ F. oxysporum: 145,8333 b } \\
\hline & R. solani: $4,1100 \mathrm{a}$ & \multicolumn{2}{|c|}{ R. solani: 272,4662 a } & \multicolumn{2}{|c|}{ R. solani: $87,0700 \mathrm{a}$} \\
\hline
\end{tabular}

df - degrees of freedom; SR2 - Streptomyces xanthochromogenes; SR4 - Streptomyces anulatus isolated from rhizosphere of Scots pine; SG9 Streptomyces cyaneus isolated from bulk soil of Scots pine; mean values marked with the same letter do not differ significantly $(p \leq 0,05)$ 
lated with this fungus had significant inhibition of root and shoot growth in seedlings cultivated both in sterile and non-sterile soil (70\% dead seedlings).

About $30-80 \%$ seedlings cultivated in sterile soil survived after inoculation with $R$. solani and different actinomycetes strains.

In non-sterile soil the positive effect of actinomycete protection was even more pronounced. The percentage of live seedlings was $70-90 \%$. Among the analyzed actinomycetes, the most favorable biocontrol interactions both in sterile and non-sterile soil were found in Streptomyces cyaneus (strain SG9) isolated from root free soil of the pine (Table 2).

No pathogenic effects of Fusarium oxysporum was observed in pine seedlings. Seedling growth parameters (both in sterile and non-sterile soil) after inoculation with this fungus were similar to control.

Growth substrate sterility was important for the condition of the seedlings. Cultured seedlings in non-sterile soil were characterized by a statistically different elongation and mass of the shoot, whereas in sterile soil better development of the seedling root system was observed.

Inoculation of the seedlings with only actinomycetes had no negative effects on the growth of pine seedlings, while the positive effect was small. In general all actinomycetes elongated the root to a small extent, and one of the strains of Streptomyces anulatus (SR4) additionally increased the shoot mass (Table 3,4 ).

\section{Discussion}

The ability of actinomycetes to produce antibiotics with a differentiated spectrum of activity and biological specificity is commonly known. Practical application of these secondary metabolites is not limited to medicine and veterinary medicine. These compounds are also used in phytopathology and in combat with plant pathogens, albeit uncommonly (Saadoun and Gharaibeh, 2003; Davelos et al. 2004; Oskay et al. 2004).

Metabolites released into the environment play an important role in shaping the interactions between microorganisms (Chmiel 1982; Dahm et al. 1986; Marcinowska 1993) and maintaining biological equilibrium in natural environments (Bressan 2003; Tian et al. 2004; Aghighi et al. 2004; Zaitlin et al. 2004).

Our investigations on the interactions between microorganisms, similar to those of other authors (Chmiel 1982; Shoda 2000) indicate that these interactions may be inhibitory, stimulating, synergistic or neutral. The inhibitory effect of actinomycetes on other microorganisms is mainly ascribed to antibiotics, while other aspects of actinomycetes metabolic activity also play an important role in these processes.

The stimulating effect of actinomycetes on other microorganisms in the environment may be linked to the formation and secretion of metabolites such as amino acids, sugars, organic acids and vitamins into the environment.

According to some scientists the site of intense synthesis of antibiotic compounds is bulk soil, even though greater competition for space and nutrients occurs in the rhizosphere of plants (Shirokikh et al. 2002). Therefore other researchers believe that microorganisms find particularly appropriate conditions for antibiotic synthesis in the plant rhizosphere (Crawford et al.1993; Marcinowska 1993; Sturz et al. 1997). Antibiotic production being highly regulated, is belived to be produced under starvation conditions when the colony is ready for sporulation and not in a nutrient-rich environment (Hopwood 2007). Aerial mycelium of Streptomyces colonies develops at the expense of nutrients obtained from the decomposition of the substrate mycelium. The released metabolites (sugars, amino acids and others) may be attractants for many microorganisms and the antibiotics produced by actinomycetes may act in this way at small distances (Hopwood 2007).

The results of our investigations are similar to the observations of Shirokikh et al. (2002) and indicate that the environment with the highest number of actinomycetes antagonistic to pathogenic fungi is root-free soil and that strains isolated from the rhizosphere are characterized by a weaker antagonism. However, the investigations of Crawford et al. (1993) have shown that actinomycetes isolated from bulk soil and the rhizosphere demonstrated a similar degree of inhibition of the pathogenic fungus Pythium ultimum.

Investigations in vitro on the antagonistic interactions of actinomycetes and pathogenic fungi performed by various research groups often differ in the type of culture medium used, the temperature of the culture or the method of inoculating the media (Crawford et al. 1993; Yuan and Crawford 1995; Trejo-Estrada et al. 1998; Getha and Vikineswary 2002). Such factors may affect the results obtained in these investigations.

In our investigations two methods were used to determine the mutual interactions of actinomycetes and fungi: according to Yuan and Crawford (1995) and according to Aghighi et al. (2004), differing in the time of inoculation of the fungus and the actinomycetes. Different or similar results were obtained depending on the analyzed actinomycetes strain. With simultaneous inoculation of the medium with the fungus and Streptomyces varsoviensis (SG5) a strong antagonism of the actinomycetes was observed, whereas when the fungus was inoculated into a 5-day actinomycete culture the interaction was much weaker. Some actinomycetes (Streptoverticillium olivovertici (SG3) and Streptomyces griseoviridis (SG8)) inhibited fungal growth to a similar extent regardless of the time of inoculation of the medium with the fungus. 
A significant effect of the time of culture on the degree of actinomycete antagonism was also demonstrated by the investigations of Yuan and Crawford (1995). The antagonism of Streptomyces lydicus WYEC108 to different species of pathogenic fungi increased with the time of duration of the mixed cultures and was stronger on the 5 th than on the 2 nd day of mixed culture.

Few investigations have addressed the effect of exopolysaccharides produced by actinomycetes on fungi. In this work the inhibitory action of exopolysaccharides was only demonstrated against fungi from the genus Fusarium. Strains of Rhizoctonia solani were resistant to the action of these compounds.

In the antagonism of actinomycetes against fungi an important role is played by chitinases, enzymes which hydrolyze their cell wall. Many authors have shown a relationship between chitinolytic activity of actinomycetes and their antifungal activity (Mahadevan and Crawford 1997; de Boer et al. 1998; Hoster 2005). Comparison of the relationship between chitinolytic activity of actinomycetes analyzed by us (Golińska and Dahm 2011a) and the observed degree of inhibition of fungi in mixed culture has shown that these parameters were not always correlated, e.g. Streptomyces cyaneus (SG9) and Streptomyces anulatus (SR4) did not produce chitinases in the conditions of the experiments performed or showed a low activity of these enzymes, but in cultures with fungi strongly inhibited their growth. Experiments performed by de Boer et al. (1998) have also shown that the growth of fungi was inhibited both by chitinolytic microorganisms and by those which were incapable of the synthesis of such enzymes. However, in other analyzed strains there was a correlation between the degree of fungal inhibition and the chitinolytic activity of actinomycetes. A high chitinolytic activity and a strong antagonism against fungi were found in the actinomycetes Streptomyces griseoviridis (SG1), S. varsoviensis (SG5), S. griseoviridis (SG8). These observations correspond to the conclusions of Hoster et al. (2005), who demonstrated a high correlation between the chitinolytic activity of various strains of Streptomyces and the degree of their antagonism against pathogenic fungi. For the biological control of diseases caused by Phytophthora infestans, Streptomyces violaceusniger (strain YCED-9), was selected, a strong antagonist in vitro of this strong fungal pathogen. The antagonism of this strain was not linked with chitinolytic activity but with antibiotic synthesis. This one strain may produce several compounds with antibiotic character: AFA (with activity against Fusarium), a fungicidal polyene complex similar to guanidylfungin A or nigericin and geldanamycin, strongly inhibiting the growth of the fungi Pythium and Phytophthora spp.
The success of biocontrol depends to a large extent on the basic knowledge on the subject of the selected antagonist (Bressan 2003). In general, microorganisms as potential biocontrol factors are selected in in vitro tests analysing their antagonism against pathogens. After the analysis of our results we selected three strains of actinomycetes (Streptomyces xanthochromogenes (SR2), S. anulatus (SR4) and S. cyaneus (SG9)) showing a strong antagonism to the analysed pathogenic fungi and used them in investigations of respective interactions of actinomycetes and pathogenic fungi on the roots of pine seedlings in greenhouse experiments.

The control of plant pathogens using biological factors is still not applied on a large scale but many investigations have shown the large possibilities of using this method (Bressan 2003).

Most investigations on biocontrol concentrate on pathogens and microorganisms which combat those of them which are associated with cultivated plants (rice, maize, tomato, tobacco etc.) - this is understandable from the economic point of view. However, the search for biological factors as an alternative to chemical compounds which combat forest tree pathogens (especially in forest nurseries) is also important (Dumroese et al. 1998; Rudawska 2000).

The observations performed by us in greenhouse experiments have shown a role for actinomycetes in protecting pine seedlings against Rhizoctonia solani.

The best biocontrol organism was the strain Streptomyces cyaneus (SG9), isolated from free root soil. It protected seedlings grown in both sterile and non-sterile soil against infection by this pathogen. The rhizosphere actinomycetes Streptomyces xanthochromogenes and Streptomyces anulatus showed a lower biocontrol ability and only in non-sterile soil.

The hypothesis is probable that plant pathogens are effectively inhibited by mixed groups of antagonists. Different microorganism populations may indicate differentiated mechanisms alleviating or fully reducing the effects of plant diseases, as well as the possibility of their occurrence (Raupach and Kloepper 1998).

Fusarium oxysporum was found to be non-pathogenic for pine seedlings even though it was obtained from a collection of strong pathogens. Fungi may lose pathogenicity among others after successive passages (Mańka 1992). The growth of seedlings inoculated with $F$. oxysporum did not differ significantly from the growth of control seedlings, and moreover inoculation of the seedlings with an actinomycete (especially Streptomyces anulatus isolated from the rhizosphere) significantly stimulated their growth.

Berg et al. (2005) moreover found that non-pathogenic or weakly pathogenic strains of well-known species of pathogenic fungi (among others Fusarium 
oxysporum) may be antagonists of other pathogenic fungi e.g. Verticillium dahliae Kleb.

\section{Acknowledgements}

This research has been financed by a grant from Polish Ministry of Science and Higher Education (Grant No. 2 PO4 C 040 29) and grant of Nicolaus Copernicus University (Grant No. 304-B/2010).

\section{References}

Aghighi S., Shahidi Bojar G.H., Rawashdeh R., Batayneh S., Saadoun J., 2004. First report of antifungal spectra of activity of Iranian Actinomycetes strains against Alternaria solani, Alternaria alternata, Fusarium solani, Phythophthora megasperma, Verticillium dahliae and Saccharomyces cerevisiae. Asian Journal of Plant Sciences 3: 463-471.

Barabasz W., Vořišek K. 2002. Biodiversity of microorganisms in soil environments. In: Microbial activity in diverse environments. Barabasz W. (ed.). Kraków: Akademia Rolnicza, pp. 23-34. (In Polish with English Summary)

Berg G., Zachow Ch., Lottmann J., Gõtz M., Costa R., Smalla K. 2005. Impact of plant species and site on rhizosphere - associated fungi antagonistic to Verticillium dahliae Kleb. Applied and Environmental Microbiology 71: 4203-4213.

Bressan W. 2003. Biological control of maize seed pathogenic fungi by use of actinomycetes. BioControl 48: 233-240.

Chattopadhyay S.K., Nandi B. 1982. Inhibition of Helminthosporium oryzae and Alternaria solani by Streptomyces longisporus (Krasil' nikov) Waksman. Plant and Soil 69: 171-175.

Chmiel A. 1982. Why do microorganisms produce antibiotics? Postępy Mikrobiologii 21: 211-234. (In Polish with English Summary).

Coombs J.T., Michelsen P.P, Franco C.M.M. 2004. Evaluation of endophytic actinobacteria as antagonists of Gaeumannomyces graminis var tritici in wheat. Biological Control 29: 359-366.

Crawford D.L. 1988. Biodegradation of agricultural and urban wastes. In: Actinomycetes in Biotechnology. In: Goodfellow M., Williams S.T., Modarski M. (eds). London: Academic Press, pp. 433-459.

Crawford D.L., Lynch J.M., Whipps J.M., Ousley M.A. 1993. Isolation and characterization of actinomycete antagonists of fungal root pathogen. Applied and Environmental Microbiology 59: 3899-3905.

Dahm H., Różycki H., Strzelczyk, E. 1986. Bacteria and actinomycetes of the soil and the root zone of forest trees. Postępy Mikrobiologii 25: 103-120. (In Polish with English Summary).
Davelos A.L., Kinkiel L.L., Samac D.A. 2004. Spatial variation in frequency and intensity of antibiotic interactions among Streptomycetes from prairie soil. Applied and Environmental Microbiology 70: 1051-1058.

de Boer M., Boom P., Kindt F., Keurentjes J.J.B., Van der Sluis I., Van Loon L.C., Bakker P.A.H.M. 2003. Control of Fusarium wilt of radish by combining Pseudomonas putida strains that have different disease-suppressive mechanisms. Phytopathology 93: 626-632.

de Boer W., Gunneweik P.J.A., Lafeber P., Janse J.D., Spit B.E., Woldendorp J.W. 1998. Anti-fungal properties of chitinolytic dune soil bacteria. Soil Biology and Biochemistry 30: 193-203.

Dong H.Z, Li W.J., Zhang D.M., Tang W. 2003. Differential expression of induced resistance by an aqueous extract of killed Penicillium chrysogenum against Verticillium wilt of cotton. Crop Protection 22: 129-134.

Dumroese R.K., Jamek R.L., Wenny D.L. 1998. Interactions among Streptomyces griseoviridis, Fusarium root disease, and Douglas-fir seedlings. New Forests 15: 181-191.

Fravel D.R., Roberts D.P.1991. In situ evidence for the role of glucose oxidase in the biocontrol of Verticillium wilt by Talaromyces flavus. Biocontrol Science and Technology 1: 91-99.

Getha K., Vikineswary S. 2002. Antagonistic effects of Streptomyces violaceusniger strain G10 on Fusarium oxysporum f. sp. cubense race 4: Indirect evidence for the role of antibiosis in the antagonistic process. Journal of Industrial Microbiology and Biotechnology 28: 303-310.

Gohar Y., Beshay U., Daba A., Hafez E. 2006. Bioactive compounds from Streptomyces nasri and its mutants with special reference to proteopolysaccharides. Polish Journal of Microbiology 55: 179-187.

Golińska P., Dahm H. 2011a. Enzymatic activity of actinomycetes from the genus Streptomyces isolated from the bulk soil and rhizosphere of the Pinus sylvestris. Dendrobiology 65: 37-46.

Golińska P., Dahm H. 2011b. Occurrence of actinomycetes in forest soil. Dendrobiology 66: 3-13.

Hopwood D.A. 2007. Streptomyces in nature and medicine. The antibiotic makers. New York: Oxford University Press.

Hoster F., Schmitz J.E., Daniel R. 2005. Enrichment of chitinolytic microorganisms: isolation and characterization of a chitinase exhibiting antifungal activity against phytopathogenic fungi from a novel Streptomyces strain. Applied Microbiology and Biotechnology 66: 434-442.

Kämpfer P. 2006. The Family Streptomycetaceae, Part I: Taxonomy. In: The Prokaryotes. Vol. 3: Archaea. Bacteria: Firmicutes, Actinomycetes. Dworkin, 
M., Falkow, S., Rosenberg, E., Schleifer K-H., Stackebrandt E. (eds). New York: Springer, pp. 538-604.

Kucuk C., Kivanc M. 2003. Isolation of Trichoderma spp. and determination of their antifungal, biochemical and physiological features. Turkish Journal of Biology 27: 247-253.

Kutzner H.J. 1981. The Family Streptomycetaceae. In: The Prokaryotes. A Handbook on Habitats Isolation and Identyfication of Bacteria. Starr, M.P., Stalp H., Truper H.G., Balows A., Schlegel H.G., (eds). Berlin Heidelberg, New York: Springer-Verlag, pp. 2028-2090.

Lechevalier M.P. 1988. Actinomycetes in agriculture and forestry. In: Actinomycetes in Biotechnology. Goodfellow M., Williams S.T., Modarski M., (eds). San Diego: Academic Press, pp. 327-358.

Mahadevan B., Crawford D.L. 1997. Properties of the chitinase of the antifungal biocontrol agent Streptomyces lydicus WYEC108. Enzyme and Microbial Technology 20: 489-493.

Mańka K. 1992. Forest phytopathology. Warszawa: PWRiL. (In Polish).

Marcinowska K. 1993. Occurrence of antagonistic actinomycetes in soils of mountain grassland ecosystems. Studia Ośrodka Dokumentacji Fotograficznej 22: 161-179. (In Polish with English Summary).

Marcinowska K. 2002. Properties, occurrence and significance of actinomycetes in nature. In: Microbial activity in diverse environments. Barabasz W. (ed). Kraków: Akademia Rolnicza, pp. 121-130. (In Polish with English Summary).

McCarthy A.J. 1987. Lignocellulose degrading actinomycetes. FEMS Microbiology Reviews 46: 145-163.

McCarthy A.J., Williams S.T. 1992. Actinomycetes as agents of biodegradation in the environment - a reviev. Gene 115: 189-192.

Oskay M., Tamer A., Azeri C. 2004. Antibacterial activity of some actinomycetes isolated from farming soils of Turkey. African Journal of Biotechnology 3: 441-446.

Paul E.A., Clark F.E. 2000. Soil microbiology and biochemistry. Lublin: UMCS. (In Polish).

Raupach G.S., Kloepper J.W. 1998. Mixtures of plant growth promoting rhizobacteria enhance biological control of multiple cucumber pathogens. Phytopathology 88: 1158-1164.

Rudawska M. 2000. Significance of ectomycorrhizae in biological preservation of forest trees against soil pathogens. Sylwan 4: 27-38. (In Polish).

Russel S. 1977. Antibiotics. Warszawa: PWN. (In Polish).

Saadoun I., Gharaibeh R. 2003. The streptomyces flora of Badia region of Jordan and its potential as a source of antibiotics active against antibiotic-resistant bacteria. Journal of Arid Environments 53: 365-371.

Shirling E.B., Gottlieb D. 1966. Methods for characterization of Streptomyces species. International Journal of Systematic Bacteriology 16: 313-340.

Shirokikh I.G., Zenova G.M., Zvyaginstev, D.G. 2002. Actinomycetes in the rhizosphere of barley grown on acid soddy podzolic soil. Microbiology (Moscow) 71: 455-459.

Shoda M. 2000. Bacterial control of plant diseases. Journal of Bioscience and Bioengineering 89: $515-521$.

Sturz A.V., Carter M.R., Johnston H.W. 1997. A review of plant disease, pathogen interactions and microbial antagonism under conservation tillage in temperate humid agriculture. Soil and Tillage Research 41: 169-189.

Tian X.L., Cao L.X., Tan H.M., Zeng Q.G., Jia Y.Y., Han W.Q., Zhou S.N. 2004. Study on the communities of endophytic fungi and endophytic actinomycetes from rice and their antipathogenic activities in vitro. World Journal of Microbiology and Biotechnology 20: 303-309.

Trejo-Estrada S.R., Paszczyński A., Crawford D.L. 1998. Antibiotics and enzymes produced by the biocontrol agent Streptomyces violaceusniger YCED-9. Journal of Industrial Microbiology and Biotechnology 21: 81-90.

Whipps J.M. 2001. Microbial interactions and biocontrol in the rhizosphere. Journal of Experimental Botany 52: 487-511.

Williams S.T., Goodfellow M., Alderson G., Wellington E.M.H., Sneath P.H.A., Sackin M.J. 1983. Numerical classification of Streptomyces and related genera. Journal of General Microbiology 129: 1743-1813.

Yuan W., Crawford D.L. 1995. Characterization of Streptomyces lydicus WYEC108 as a potential biocontrol agent against fungal root and seed rots. Applied Environmental Microbiology 61: 3119-3128.

Zaitlin B., Turkington K., Parkinson D., Clayton G. 2004. Effects of tillage and inorganic fertilizers on culturable soil actinomycete communities and inhibion on fungi by specific actinomycetes. Applied Soil Ecology 26: 53-62.

Zakalyukina Y.V., Zenova G.M., Zvyagintsev D.G. 2002. Acidophilic soil actinomycetes. Microbiology (Moscow) 71: 342-345.

Zakalyukina Y.V., Zenova G.M., Zvyagintsev D.G. 2004. Peculiarities of growth and morphological differentiation of acidophilic and neutrophilic soil streptomycetes. Microbiology (Moscow) 73: 74-78. 